\title{
Nutrition, oxidative stress and intestinal dysbiosis: Influence of diet on gut microbiota in inflammatory bowel diseases
}

\author{
Giovanni Tomaselloa,b,c,e, Margherita Mazzola ${ }^{\mathrm{a}}$, Angelo Leone', Emanuele Sinagra ${ }^{\mathrm{d}, \mathrm{e}}$, Giovanni Zummo ${ }^{\mathrm{a}}$, Felicia Farina ${ }^{\mathrm{a}}$, \\ Provvidenza Damianic, Francesco Cappello a, , , Alice Gerges Geagea ${ }^{\text {a, }}$, Abdo Jurjus ${ }^{\mathrm{f}}$, Tarek Bou Assi ${ }^{\mathrm{g}}$, Massimiliano Messinah \\ Francesco Carinia,c
}

\begin{abstract}
Background. Microbiota refers to the population of microorganisms (bacteria, viruses and fungi) that inhabit the entire gastrointestinal tract, more particularly the colon whose role is to maintain the integrity of the intestinal mucosa and control the proliferation of pathogenic bacteria. Alteration in the composition of the gut microbiota is called dysbiosis. Dysbiosis redisposes to inflammatory bowel diseases such as ulcerative colitis, Crohn disease and indeterminate colitis. Methods. The purpose of this literature review is to elucidate the influence of diet on the composition of the gastrointestinal microbiota in the healthy gut and the role of diet in the development of dysbiosis.

Conclusion. The "Western diet", in particular a low - fiber high fat/high carbohydrate diet is one factor that can lead to severe dysbiosis. In contrast, "mediterranean" and vegetarian diets that includes abundant fruits, vegetables, olive oil and oily fish are known for their anti-inflammatory effects and could prevent dysbiosis and subsequent inflammatory bowel disease.
\end{abstract}

Key words: inflammatory bowel diseases, colorectal cancer, intestinal dysbiosis, gut microbiota, healthy diet

Received: May 12, 2016; Accepted with revision: September 27, 2016; Available online: October 26, 2016

http://dx.doi.org/10.5507/bp.2016.052

a'Department of Experimental Biomedicine and Clinical Neuroscience, Section of Human Anatomy, (BIONEC), University of Palermo, Italy
'SChool of Medicine and Surgery - Hypathia Course, University of Palermo, Italy
'AOUP "P. Giaccone", School of Medicine and Surgery, University of Palermo, Italy
'Fondazione Istituto S. Raffaele - G. Giglio, Gastroenterology and Endoscopy Unit, Cefalu, Italy
'Euro-Mediterranean Institute of Science and Technology (IEMEST), Palermo, Italy
'Department of Anatomy, Cell Biology and Physiological Sciences, Faculty of Medicine, American University of Beirut (AUB), Beirut, Lebanon
'Department of Laboratory Medicine, Psychiatric Hospital of the Cross, Jal Eddib, Lebanon
'Department of Radiologic Science, School of Medicine and Surgery, University of Palermo, Italy
'Department of Experimental Biomedicine and Clinical Neuroscience, Section of Histology, (BIONEC), University of Palermo, Italy
Corresponding author: Margherita Mazzola, e-mail: margheritamazzola@hotmail.it

\section{INTRODUCTION}

Many areas of current research focus on the the influence and effects of dietary habits on human health, especially in Western countries, where he largest proportions of so- called "junk food" consumers reside. The "western diet", mainly refined carbohydrates, simple sugars and saturated fats, is linked to various metabolic disorders.

The purpose of this review is to illustrate how dietary habits can influence the composition of the intestinal microbiota leading to dysbiosis and inflammatory bowel disease (IBD). After a brief overview of the gut anatomy and the role of the microbiota in healthy gut, this review depicts how the microbiota composition varies based on diet and how this in turn may lead to the onset of systemic metabolic disorders and/or IBD.

\section{GUT MORPHOLOGY}

The habitat of the gut microbiota is represented by the intestine. The latter consists of two major portions: the small intestine, divided into duodenum, jejunum and ileum responsible for digestion and nutrient absorption and the large intestine, divided into cecum, colon and rectum is responsible for water absorption and formation of feces. The intestinal wall is composed of four superimposed layers: from the outermost layer, we find the serosa, the muscularis propria, the submucosa and finally the mucosa. The small intestine has villi created mainly in enterocytes which show on their surface, protrusions called microvilli. These protrusions increase the absorption surface and thus facilitate the digestive process and the absorption of nutrients. The large intestine epithelium comprises colonocytes similar to the enterocytes. However, their main function is to absorb water. Colonocytes are arranged to form along goblet cells, simple tubular glands which secrete neutral mucus to lubricate the intestinal contents. The highest concentration of bacteria is found in the large intestine. For a more detailed description, please refer to specialized books ${ }^{1,2}$. 


\section{MICROBIOTA}

In the human gut, there are trillions of microorganisms which together are known as microbiota ${ }^{3,4}$. Intestinal bacteria have the highest concentration, approximately 100 trillion, with a density of $9 \times 10^{13-14}$ and considerable biodiversity (approximately 1000 bacterial species). In the colon, there are around 160 to 500 different bacterial species with a wide range of specific microbiological characteristics $^{4.7}$.

The total microbiota genome is around 100 times greater in size than the human genome and is defined by the term microbiome ${ }^{7}$. During pregnancy, the fetal gut is sterile and therefore bacterial colonization and the acquisition of the microbiota occur at birth. During the passage through the birth canal, the neonatal intestine is colonized by the maternal, anal or vaginal flora that contain bacteria such as Bacteroidetes, Bifidobacterium, Prevotella and Lactobacillus spp. ${ }^{5,8}$. However, for babies delivered by cesarean section, intestinal colonization occurs mainly by bacteria from the skin and from the hospital environment ${ }^{8}$.

As long as babies are breastfed, gut microbiota consists primarily of Bifidobacterium spp. This composition changes with the introduction of solid foods until they reach the final composition seen in adulthood ${ }^{8}$. However, in adults, the microbiota varies in number and species based on the location in the gastrointestinal tract. In the stomach, several microorganisms are present mainly Lactobacillus, Streptococcus, Helicobacter Pylori, Peptostreptococcus and Candida. In the duodenum, jeju- num and proximal ileum there are mainly Lactobacillus and Streptococcus, while the distal ileum contains around $10^{7}$ to $10^{8}$ bacteria including Streptococcus, Bacteroidetes, Actinomycinae, Corynebacteria and Clostridium. On the other hand, the colon contains around $10^{11}$ to $10^{12}$ bacteria mainly Clostridium Type IV and XIV, Bacteroidetes, Bifidobacterium and Enterobacteriaceae ${ }^{9}$. As a result, it is clear that most bacteria reside in the colon and they are divided into four main groups: Firmicutes, represents alone $64 \%$ of the microbiota; Bacteroidetes (23\%); followed by Actinobacteria and Proteobacteria9.

The microbiota plays a vital role in the health of the host. In fact, it controls the proliferation of pathogenic bacteria present in the intestinal tract (such as Clostridia or Colibacillacea) (ref. ${ }^{5,10}$ ), stimulates the immune system, regulates the absorption of nutrients ${ }^{11}$, regulates the host metabolism and physiology ${ }^{12}$, intervenes in the production of vitamins and enzymes such as vitamin $\mathrm{K}$ and biotin ${ }^{6}$, and in the synthesis of compounds useful for the trophism of colonic mucosa and required for cell renewal ${ }^{5,10}$.

Within the gut, the microbiota plays different roles, including the fermentation of amino acids and saccharides, with the production of short-chain fatty acids (SCFAs), succinate, ethanol, $\mathrm{H}_{2}$, amines, lactate, phenols, thiols and indoles, disposal of hydrogen (as acetate $\mathrm{H}_{2} \mathrm{~S}$ and methane) degradation of undigested proteins and carbohydrates, and the transformation of bile acids ${ }^{8}$. Actually, the production of SCFAs, including butyrate, propionate and acetate, plays an essential role in maintaining a healthy mucosa and the production of anti-inflammatory interleukins ${ }^{5,11}$. In brief, the SCFAs are derived from bacte-

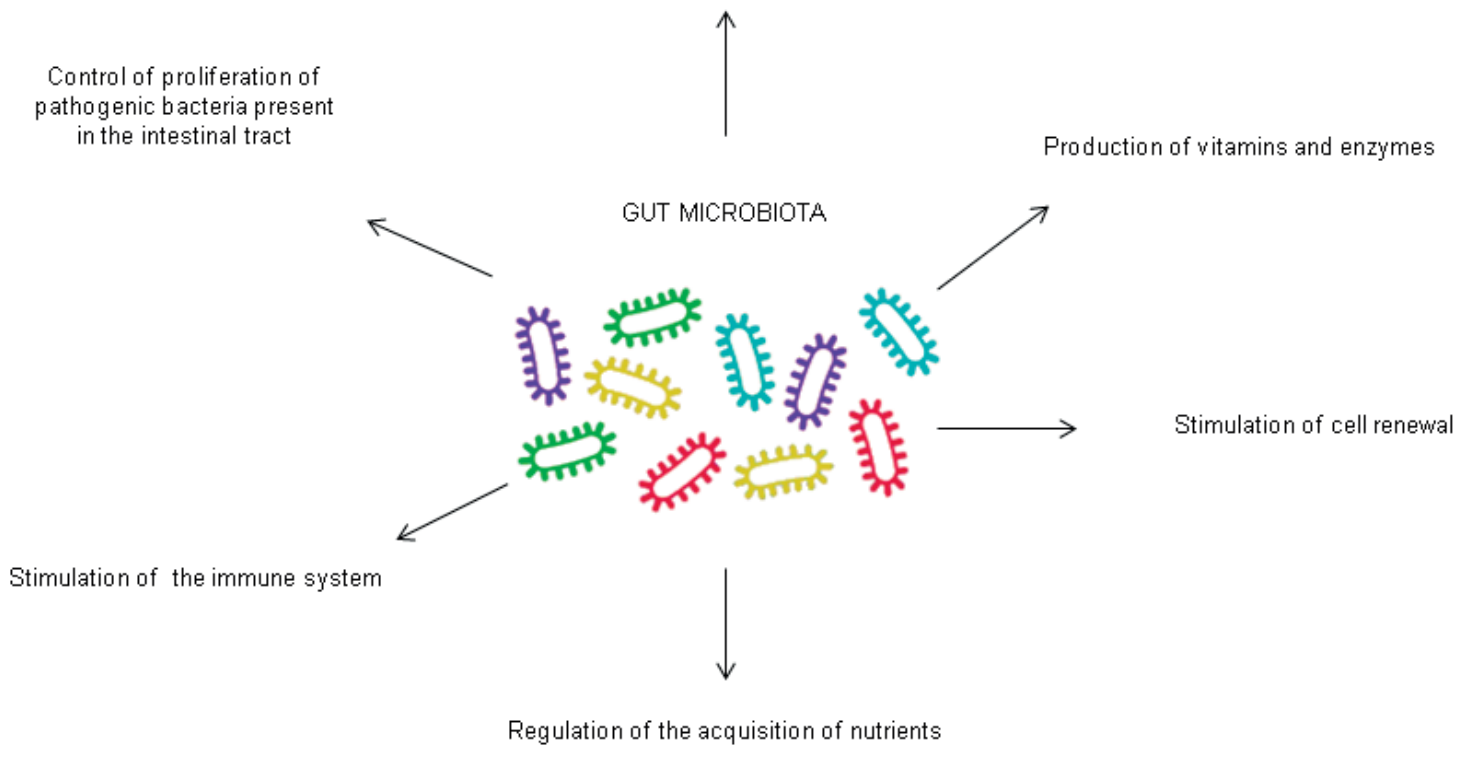

Fig. 1. Role of gut microbiota in humans. Human gut microbiota plays roles: stimulates the immune systém and cell reneval, produces vitamins and enzymes (such as vitamin $\mathrm{K}$ and biotin), enhances the synthesis of compounds useful for the trophism of colonic mucosa, and regulates the absorption of nutrients. One of its most important functions, however, is to regulate and influence the host's metabolism and to control the proliferation of pathogenic bacteria found in the gastrointestinal tract (such as Clostridia or Colibacillacea). 
rial fermentation of dietary carbohydrates. This fermentation process occurs in the colon by Lactobacilli and Bifidobacteria under anaerobic conditions ${ }^{5}$.

Moreover, butyric acid is the preferred source of energy for colonocytes. Butyric acid has an important anti-inflammatory effect and controls the proliferation, differentiation and apoptosis of colonocytes. In addition, it strengthens the defensive barrier of the colon by increasing the production of mucin and antimicrobial peptides and by decreasing the intestinal epithelial permeability and increasing the expression of tight junction's proteins ${ }^{5}$.

Another important function of the microbiota is to keep the concentration of pathogens within limits. This action is accomplished via various mechanisms including direct competition for nutrients, increase of the mucus layer on the intestinal mucosa and through the development and the stimulation of the immune system, particularly of the gut associated lymphatic tissue (GALT) (ref. ${ }^{13}$ ).

\section{DYSBIOSIS AND RELATED DISEASES}

Any alteration of bowel eubiosis or in the composition of the microbiota is called dysbiosis. Actually, the composition of the microbiota depends on several factors including the structure of the host's intestinal epithelium, peristalsis, dietary changes, age, genes, temperature, interaction between different bacterial species, response of the immune system in particular T and B cells, administration of antibiotics or radiation and chemotherapy drugs, environmental, physical and finally psychological stress ${ }^{5,6,14}$.
Consequently, dysbiosis causes an alteration of the intercellular tight junctions that are responsible for keeping the integrity of the intestinal mucosa ${ }^{15}$ and its permeability which is crucial to prevent the access of pathogens ${ }^{5}$. Otherwise there is activation of the MALT (Mucosal Associated Lymphatic Tissue) with activation of the inflammatory cascade (leukocytes, cytokines, TNF- $\alpha$ ) and consequently massive tissue damage ${ }^{15}$.

Dysbiosis is also related to the development of a number of diseases including type 2 diabetes, allergies, fatty liver disease, obesity and IBD among others ${ }^{7}$.

IBD, such as Crohn's disease (CD), ulcerative colitis (UC) and Indeterminate Colitis, are chronic and progressive immune disorders related to alterations in (i) the composition of the microbiota (dysbiosis), (ii) immune response (iii) genetic and (iv) environmental factors ${ }^{10,16}$. It is estimated that there are 3.7 million people living with IBD in American and European populations ${ }^{16}$.

$\mathrm{CD}$ is an IBD that affects the entire gastrointestinal tract, from the mouth to the anus, with possible alternations in all four layers of the intestineand possible anal stenosis, fistulas, and/or abscesses. It is manifested by symptoms that include periods of remission and disease flare. On the other hand, UC is an IBD that affects the terminal portion of the gastrointestinal system, rectum and colon and, characterized by a hyperemic mucosa, exudate and erosions ${ }^{15-17}$.

The pathogenesis of IBD is still unclear. It is defined as a multifactorial disease. Contributing factors range from genetic (i.e. gene NOD2), to changes of microbiota composition, exposure to environmental stresses such as

\section{CAUSES}

Interaction between differentbacterial species

Dietary changes

Genetic features

Genetic features
Physical and psychological stress
Antibiotics
Radiation and chemotherapy

Response of the immune system

\section{DISEASES}

Type 2 diabetes

Inflammatory bowel diseases (IBD)

Fatty liver disease

Allergies

Colorectal can cer (CRC)

\section{Dysbiosis}

Obesity

Fig. 2. Causes of dysbiosis and related diseases. Dysbiosis is a state of imbalance between the various organisms inhabiting the human gut. It can be caused by hereditary factors, some drugs such as antibiotics, radiotherapy and chemotherapy, age, but is mostly related to poor nutrition and physical and psychological stress which results in a dysregulation of the immune system. All of these predispose to the onset of conditions as type 2 diabetes, liver disease and especially inflammatory bowel disease (IBD) and colorectal cancer (CRC). 
cigarette smoking, diet, physical and psychological stress, surgery (i.e. appendectomy), sedentary lifestyle, personal hygiene and use of drugs ${ }^{6,16,18}$.

Another possible etiology of IBD is through oxidative stress which is characterized by the presence of reactive oxygen species (ROS). ROS (such as hydrogen peroxide, hydroxyl radical, superoxide anion and peroxynitrite) arise from incomplete reduction of oxygen ${ }^{19}$ and are often correlated with dysbiosis. It is believed that oxidative stress induced by ROS increases the inflammatory reaction leading, probably through a positive feedback mechanism, to an increased production of ROS and subsequent tissue damage ${ }^{20}$.

Several studies have shown a correlation between increased oxidative stress and a Western-style diet rich in fats and refined sugars. These products in fact, if taken frequently and in large quantities, cause an increase of the inflammatory state with ROS production and subsequent amplification of the inflammatory cascade ${ }^{21-23}$.

Currently, there are also discussions about the potential role of heat shock proteins (HSPs) in the pathogenesis of IBD. HSPs are involved in various processes such as folding, translocation and degradation of intracellular proteins under normal and stressful conditions. Being highly conserved molecules with similar sequences in bacterial and human orthologs (molecular mimicry),
HSPs can stimulate an immune response, both innate and adaptive and hence primary targets of the autoimmune response $^{5,21}$. Furthermore, intestinal dysbiosis is often accompanied by a reduction in obligate anaerobic bacteria and an increase in facultative anaerobes. A comparison between the resident gut microbiota in IBD patients with the microbiota of healthy subjects has shown that in IBD patients, there are fewer f phylum Firmicutes and a higher load of Enterobacteriaceae including E. coli ${ }^{4}$. Another study showed that CD patients had a decreased number of Faecalibacterium prausnitzii (Firmicutes) responsible for the production of butyric acid ${ }^{16}$.

\section{HOW NUTRITION AND DIET CAN INFLUENCE MICROBIOTA AND CORRECT DYSBIOSIS}

It is well known that a high fat/carb diet, low in fruits and vegetables increases the risk of metabolic disorders. In addition, it is well demonstrated that diet has a great influence (estimated at $57 \%$ compared with $12 \%$ for genetic factors) on the composition of the microbiota ${ }^{10}$. Targeted studies have shown that consumption of large amounts of fat and sugar in the long run, results in a degree of dysbiosis and in a change of microbiota such as an increased number of Bacterioides spp and Ruminococcus torques ${ }^{22}$.

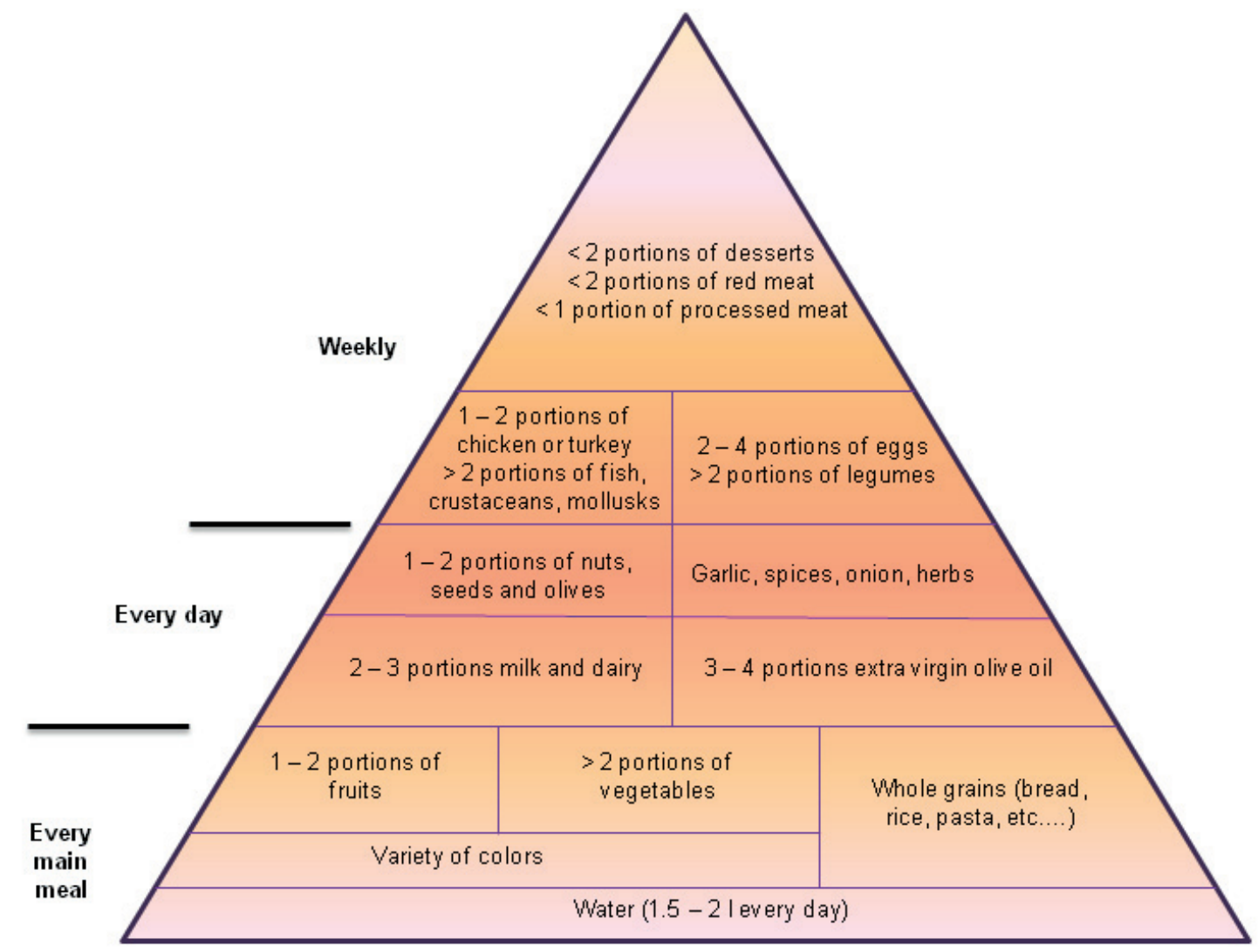

Fig. 3. Food pyramid with daily and weekly consumption of food groups. The Mediterranean food pyramid summarizes the food groups that should be eaten within a week. At the base of the pyramid, we find that water should be consumed daily in large quantities (at least 1.51 per day). Second to water, fruits, vegetables and whole grains should be consumed daily at every meal. Climbing further, we find herbs, extra virgin olive oil and dairy products, which should be consumed daily. At the top of the pyramid, we find animal products (fish, eggs and meat) and vegetables proteins, the consumption of which should be alternated and weekly. Finally, at the apex of the pyramid are the processed meats and sweets, whose consumption should be limited. 
Other studies have shown that a diet rich in saturated fatty acids and low in polyunsaturated fatty acids (derived from vegetable oils) induces dysbiosis with proliferation of Bilophila wadsworthia, leading to an inflammatory reaction mediated by Th1 cells and colitis in mice ${ }^{13,16}$.

In addition, if we compare the Western diet which is high in fat, refined carbohydrates and animal protein, to Eastern diets based on carbohydrates derived from plants, vegetables, rice and fruits, we note that the Eastern population microbiota has a higher prevalence of Prevotella spp. rather than Bacteriodes spp. compared to the Western population ${ }^{8,16}$. Furthermore, animal sources of protein and fat are associated with a greater number of Bacteriodes spp., while simple carbohydrates and fiber are mostly associated with an increase of Prevotella spp. ${ }^{8,16}$.

On the other hand, while the bacterial fermentation of carbohydrates produces SCFAs that maintains a healthy intestine, the fermentation of protein residues produces metabolites such as organic acids, phenolic compounds, indoles and ammonia which are deleterious and toxic for the intestine ${ }^{8}$.

It is well-demonstrated that diets high in fat and/or sugar destroy the intestinal microbiota, leading to dysbiosis and increased production of endotoxins ${ }^{23}$. Dysbiosis modifies the intestinal mucosa which becomes thinner and more permeable to pathogens and antigens with consequent establishment a low-grade but persistent, inflammation $^{22}$. In contrast, a diet rich in vegetables and fibers, reduces the intestinal $\mathrm{pH}$ and prevents the growth of potential pathogenic bacteria such as strains of $E$. coli and other Enterobacteriaceae ${ }^{10}$. In brief, the literature is in support of the fact that microbiota is intimately related to food quality and that diet influences the composition of the microbiota and represents a source of luminal antigens ${ }^{15}$.

In a review in 2015, Tomasello et al. ${ }^{15}$ noted that a Western-style diet, high in refined carbohydrates, simple sugars, saturated fatty acids and omega 6 fatty acids and poor in fruits and vegetables, may be a trigger for UC and CD (ref. ${ }^{15}$ ). Similarly, according to Agus et al. ${ }^{22}$, excessive consumption of animal protein is associated with an increased risk of developing CD; while the consumption of fruit and vegetables was inversely related ${ }^{15}$. Patients with $\mathrm{CD}$ also showed a shift in the microbiota, with an increased Proteobacteria and Bifidobacteria groups, and a decreased Firmicutes ${ }^{22}$.

For UC, in addition to the large consumption of refined carbohydrates and simple sugars, the consumption of large amounts of fatty acids is also associated with an increased risk $^{17}$.

An alteration of the relationship between the various categories of polyunsaturated fatty acids (PUFA), is associated with an increased risk of CD and UC (ref. $\left.{ }^{16}\right)$.

It is also reported that Vitamin D deficiency may play a role in the pathogenesis of IBD. In mice, Ananthakrishnan ${ }^{16}$ demonstrated that a deficiency of vitamin D was associated with an increased risk of colitis, whereas Vitamin D supplementation had an anti-inflam- matory effect in mice with colitis, due to the inhibition of pro inflammatory genes such as TNF genes ${ }^{16}$.

In addition, the intake of PUFA and conjugated linoleic acid (CLA) appear to have numerous benefits in IBD patients because they have anti-inflammatory characteris$\operatorname{tics}^{15}$. In fact, they decrease the production of interferon- $\gamma$ and prostaglandin E2 (ref. ${ }^{17}$ ) and modify the responsiveness of T cells ${ }^{15}$.

One diet pattern that is considered useful for the prevention/ improvement of intestinal diseases is the Mediterranean diet. This is characterized by a high intake of fruit and vegetables (rich in fiber, antioxidants and vitamins), olive oil and oily fish (rich in mono and polyunsaturated fatty acids), and whole grains and nuts ${ }^{15,24}$. It is based on the daily or weekly consumption of specific food groups according to the standardized food pyramid ${ }^{24}$.

Current research on probiotics, as a food supplement in addition to a mediterranean diet, showed that probiotics (such as Lactobacillus Rhamnosus) changes the composition of the microbiota, thus allowing the return to eubiosis ${ }^{5}$. Also, prebiotic foods, which contains soluble fiber, helps maintaining intestinal eubiosis ${ }^{25}$. Prebiotics, for example inulin, are metabolized by the gut microbiota to form SCFAs including butyric acid. Tralongo et al. ${ }^{5}$ showed that butyrate had a positive effect on the physiological activity of colonocytes and that it had an anti-inflammatory effect (expressed by reducing the production of pro-inflammatory factors such as Nf-kb) making it a valuable ally in the treatment of IBD symptoms ${ }^{5}$.

\section{CONCLUSION}

The role of nutrition in the prevention and improvement of IBD symptoms is widely demonstrated. Compared to the Western diet, there are clear benefits of the mediterranean or vegetarian diet rich in fruits, vegetables, fish oil, whole grains and olive oil, which provide nutrients such as vitamin D, essential fatty acids, minerals and fibers. These foods maintain a healthy intestinal microbiota preventing dysbiosis, which was deemed the basis of IBD.

However, the true role of the Western diet in the development of IBD has not yet been fully clarified and for this reason, it is advisable to undertake further studies.

The role of diet and probiotics supplementation in restoring the balance of intestinal flora and improving IBD symptoms is also well established. In addition to a healthy diet, it would be beneficial to take supplements to increase the levels of nutrients helpful in reducing inflammatory processes.

Author contribution: All authors contributed equally to preparing the manuscript.

Conflict of interest statement: The authors state that there are no conflicts of interest regarding the publication of this article. 


\section{REFERENCES}

1. Young B. Wheater's Functional Histology: A Text and Colour Atlas. London: Elsevier Health Sciences; 2006.

2. Mills SE. Histology for Pathologists. Fourth Edition. Philadelphia: Wolters Kluwer Health/Lippincott Williams \& Wilkins; 2012.

3. Sonnenburg ED, Smits SA, Tikhonov M, Higginbottom SK, Wingreen NS, Sonnenburg JL. Diet-induced extinctions in the gut microbiota compound over generations. Nature 2016;529(7585):212-5.

4. Rigottier-Gois L. Dysbiosis in inflammatory bowel diseases: the oxy gen hypothesis. ISME J 2013;7(7):1256-61.

5. Tralongo $P$, Tomasello G, Sinagra E, Damiani $P$, Leone A, Palumbo VD Giammanco M, Di Majo D, Damiani F, Abruzzo A, Bruno A, Cassata G, Cicero L, Noto M, Tomasello R, Lo Monte Al. The role of butyric acid as a protective agent against inflammatory bowel diseases. Euromediterranean Biomedical Journal 2014;9(4):24-35.

6. Parekh PJ, Balart LA, Johnson DA. The Influence of the Gut Microbiome on Obesity, Metabolic Syndrome and Gastrointestinal Disease. Clin Transl Gastroenterol 2015;18;6:e91.

7. Putignani L, Del Chierico F, Petrucca A, Vernocchi P, Dallapiccola $B$. The human gut microbiota: a dynamic interplay with the host from birth to senescence settled during childhood. Pediatr Res 2014;76(1):2-10.

8. Rajilić-Stojanović M, Jonkers DM, Salonen A, Hanevik K, Raes J, Jalanka J, de Vos WM, Manichanh C, Golic N, Enck P, Philippou E, Iraq FA, Clarke G, Spiller RC, Penders J. Intestinal microbiota and diet in IBS: causes, consequences, or epiphenomena?. Am J Gastroenterol 2015;110(2):278-87.

9. Sartor RB, and Mazmanian SK. Intestinal Microbes in Inflammatory Bowel Diseases. Am J Gastroenterol Suppl 2012;1:15-21.

10. Tomasello G, Tralongo P, Damiani P, Sinagra E, Di Trapani B, Zeenny MN, Hussein IH, Jurjus A, Leone A. Dismicrobism in inflammatory bowel disease and colorectal cancer: Changes in response of colocytes. World J Gastroenterol 2014;20(48):18121-30.

11. Kelder T, Stroeve JH, Bijlsma S, Radonjic M, Roeselers G. Correlation network analysis reveals relationships between diet-induced chang es in human gut microbiota and metabolic health. Nutr Diabetes 2014;4:e122.

12. Falcinelli S, Rodiles A, Unniappan S, Picchietti S, Gioacchini G, Merrifield DL, Carnevali O. Probiotic treatment reduces appetite and glucose level in the zebrafish model. Sci Rep 2016;6:18061.

13. Kamada N, Seo SU, Chen GY, Núñez G. Role of the gut micro biota in immunity and inflammatory disease. Nat Rev Immuno 2013;13(5):321-35.
14. Lozupone CA, Stombaugh Jl, Gordon Jl, Jansson JK, Knight R. Diversity, stability and resilience of the human gut microbiota. Nature 2012;489(7415):220-30.

15. Tomasello G, Abruzzo A, Sinagra E, Damiani P, Damiani F, Traina G, Campanella C, Rappa F, Marino Gammazza A, Noto M, Palumbo VD, Lo Monte AI. Nutrition in IBD patient's: what are the prospects? Progress in Nutrition 2015;17(2):79-86.

16. Ananthakrishnan AN. Epidemiology and risk factors for IBD. Nat Rev Gastroenterol Hepatol 2015;12(4):205-17.

17. Sinagra E, Tomasello G, Raimondo D, Rossi F, Facella T, Cappello F, Damiani P, Abruzzo A, Bruno A, Palumbo VD, Cosentino L, Cottone M, Criscuoli V, Noto M, Lo Monte Al. Nutrition, malnutrition and dietary interventions in inflammatory bowel disease. Progress in Nutrition 2014;16(2):79-89.

18. De Souza HS, Fiocchi C. Immunopathogenesis of IBD: current state of the art. Nat Rev Gastroenterol Hepatol 2016;13(1):13-27.

19. Sreevalsan $S$, Safe $S$. Reactive oxygen species and colorectal cancer Current colorectal cancer reports 2013;9(4):350-7.

20. Rezaie A, Parker RD, Abdollahi M. Oxidative stress and pathogenesis of inflammatory bowel disease: an epiphenomenon or the cause? Dig Dis Sci 2007;52(9):2015-21.

21. Bellavia M, Tomasello G, Romeo M, Damiani P, Lo Monte Al, Lozio L, Campanella C, Marino Gammazza A, Rappa F, Zummo G, Cocchi M, Conway de Macario E, Macario AJ, Cappello F. Gut microbiota imbalance and chaperoning system malfunction are central to ulcerative colitis pathogenesis and can be counteracted with specifically designed probiotics: a working hypothesis. Med Microbiol Immunol 2013;202(6):393-406.

22. Agus A, Denizot J, Thévenot J, Martinez-Medina $M$, Massier $S$, Sauvanet $\mathrm{P}$, Bernalier-Donadille $\mathrm{A}$, Denis $\mathrm{S}$, Hofman $\mathrm{P}$, Bonnet $\mathrm{R}$ Billard E, Barnich N. Western diet induces a shift in microbiota composition enhancing susceptibility to adherent-Invasive E. coli infection and intestinal inflammation. Sci Rep 2016;6:19032.

23. Zhang C, Li S, Yang L, Huang P, Li W, Wang S, Zhao G, Zhang M, Pang $X$, Yan Z, Liu Y, Zhao L. Structural modulation of gut microbiota in life-long calorie-restricted mice. Nat Commun 2013;4:2163.

24. Sáez-Almendros S, Obrador B, Bach-Faig A, Serra-Majem L. Environmental footprints of Mediterranean versus Western dietary patterns: beyond the health benefits of the Mediterranean diet. Environ Health 2013;12:118.

25. Calder PC. Omega-3 polyunsaturated fatty acids and inflammatory processes: nutrition or pharmacology? Br J Clin Pharmacol 2013;75(3):645-62. 